\title{
Certolizumab pegol plus methotrexate provides broad relief from the burden of rheumatoid arthritis: analysis of patient-reported outcomes from the RAPID 2 trial
}

\author{
Vibeke Strand, ${ }^{1}$ Josef S Smolen, ${ }^{2}$ Ronald F van Vollenhoven, ${ }^{3}$ Philip Mease, ${ }^{4}$ \\ Gerd R Burmester, ${ }^{5}$ Falk Hiepe, ${ }^{5}$ Dinesh Khanna, ${ }^{6}$ Enkeleida Nikaï, ${ }^{7}$ Geoffroy Coteur, ${ }^{8}$ \\ Michael Schiff ${ }^{9}$
}

\begin{abstract}
- Additional data
(supplementary tables and figures) are published online only. To view these files please visit the journal online (http://ard.bmj.com)

'Division of Immunology/ Rheumatology, Stanford University Portola Valley, California, USA

2Department of Rheumatology, Medical University of Vienna and Hietzing Hospital, Vienna, Austria

${ }^{3}$ Unit for Clinical Therapy Research, Inflammatory Diseases, Karolinska Institute, Stockholm, Sweden ${ }^{4}$ Swedish Medical Center, University of Washington, Seattle, Washington, USA ${ }^{5}$ Department of Rheumatology and Clinical Immunology, Humboldt University of Berlin, Charitié Hospital, Berlin, Germany

${ }^{6}$ Department of Medicine, Division of Rheumatology, David Geffen School of Medicine, University of California at Los Angeles, Los Angeles, California, USA

${ }^{7}$ Business \& Decision Life Sciences, Brussels, Belgium ${ }^{8} \mathrm{Global}$ Health Outcomes \& Access - Immunology, UCB Pharma SA, Brussels, Belgium ${ }^{9}$ Rheumatology Division, University of Colorado, Denver, Colorado, USA
\end{abstract}

\section{Correspondence to}

Vibeke Strand, Division of Immunology and

Rheumatology, Stanford University, 306 Ramona Road, Portola Valley, CA 94028, USA vstrand@stanford.edu

Accepted 23 January 2011 Published Online First 17 March 2011

\section{UNLOCKED}

This paper is freely available online under the BMJ Journals unlocked scheme, see http:// ard.bmj.com/info/unlocked.dtl

\begin{abstract}
Objective To assess the impact of certolizumab pegol (CZP) on patient-reported outcomes (PROs) in rheumatoid arthritis (RA), and to interpret these results using number needed to treat (NNT), and associations between PRO responses and longer term outcomes.

Methods A total of 619 patients with active RA were randomised to CZP 200 or 400 mg, or placebo plus methotrexate (MTX). PROs assessed included pain, patient's global assessment of disease activity (PtGA), physical function, fatigue and health-related quality of life. Treatment impact on PROs, NNT to achieve simultaneous improvements in multiple PROs and correlations between PROs were calculated. Times to onset of improvements greater than or equal to minimum clinically important differences (MCIDs) in pain as a determinant of clinical outcomes at week 24 were compared between week 6 and 12 responders, and in patients with improvements in pain $\geq \mathrm{MCID}$ at week 12 (week 12 responders/nonresponders).
\end{abstract}

Results CZP 200 and 400 mg plus MTX were associated with rapid, clinically meaningful improvements in all PROs. The NNT for subjects to report changes $\geq$ MCID in up to five PROs was two to three, and five for all six PROs (pain, PtGA, physical function, fatigue and short-form 36-item Physical and Mental Component Summary Scores). More patients with improvements $\geq \mathrm{MCID}$ in pain at week 6 than those at week 12 had lower disease activity at week 24 . Week 12 pain responders had better clinical outcomes at week 24 than non-responders.

Conclusions The data demonstrate that CZP provides broad relief from the burden of RA.

Trial registration number NCT00160602.

\section{INTRODUCTION}

With the advent of new biologic therapies in rheumatoid arthritis (RA), the goal of treatment has shifted to providing broad relief of physical, emotional and social disease burdens. To understand symptom relief more broadly, the assessment of clinically meaningful improvements using minimal clinically important differences (MCIDs) ${ }^{1}$ allows an understanding of the efficacy of a new therapy in a more meaningful way. In addition to evaluating the impact of treatment on individual patientreported outcome (PRO) measures, MCIDs can be used in number needed-to-treat (NNT) or predictive analyses to better understand the magnitude of treatment benefit. The NNT determines the number of patients that need to be treated in order to obtain the benefit of interest in one additional patient (small NNTs indicate a favourable treatment effect). ${ }^{2}$ Additionally, the predictive value of MCIDs can be evaluated by examining whether achieving improvements $\geq$ MCID earlier with active treatment result in better longer term clinical outcomes.

Certolizumab pegol (CZP), a PEGylated antitumour necrosis factor (anti-TNF) for the treatment of RA, has demonstrated efficacy and safety in three phase III trials. ${ }^{3-5}$ When administered with methotrexate (MTX), CZP provided rapid, sustained and clinically meaningful improvements in physical function, health-related quality of life (HRQoL) and all other PROs over 1 year. ${ }^{6}$ To further extend these observations, data from the Rheumatoid Arthritis PreventIon of structural Damage (RAPID) 2 trial were examined to investigate the NNT based on patients reporting improvements $\geq \mathrm{MCID}$, correlations between individual PROs and other clinical parameters, and times to onset of 'responses' (improvements $\geq \mathrm{MCID}$ ) as predictors of disease activity at week 24 .

\section{METHODS}

\section{Patients and study design}

Study methods of the RAPID 2 trial have been published previously. ${ }^{5}$ Briefly, patients with active RA (by 1987 American College of Rheumatology (ACR) classification criteria ${ }^{7}$ ) with inadequate responses to MTX therapy were randomised to receive CZP (400 mg at weeks 0,2 and 4 followed by CZP $200 \mathrm{mg}$ or $400 \mathrm{mg}$ ) plus MTX every 2 weeks, or placebo plus MTX for 24 weeks. The study (NCT00160602) was conducted in accordance with good clinical practice and the Declaration of Helsinki. Institutional review boards or ethics committees approved the protocol at each centre, and all patients provided written informed consent.

\section{Efficacy assessments}

The PROs were secondary efficacy end points in RAPID 2, and included assessments of patient's pain global assessment of disease activity (PtGA), physical function, fatigue and HRQoL. Pain and PtGA were evaluated using $100 \mathrm{~mm}$ Visual Analogue Scales (VAS). MCIDs for pain and PtGA VAS are $10 \mathrm{~mm}$ decreases from baseline. ${ }^{8-13}$ Physical function was evaluated by the Health Assessment Questionnaire-Disability Index (HAQ-DI). ${ }^{14}{ }^{15}$ MCID for the HAQ-DI is a 0.22-point decrease from baseline. 816 The Fatigue Assessment Scale 
(FAS) was used to assess fatigue/tiredness. MCID for the FAS is $10 \%$ of the scale range, corresponding to a 1-point decrease from baseline. ${ }^{9}{ }^{13} \mathrm{HRQoL}$ was evaluated using the Medical Outcomes Survey short-form 36-item (SF-36), which assesses eight domains: Physical Functioning (PF), Role Physical (RP), Bodily Pain (BP), General Health (GH), Vitality (VT), Social Functioning (SF), Role Emotional (RE) and Mental Health (MH), scored from 0 to $100 .{ }^{17}$ Normalised and z-transformed domain scores are grouped into Physical and Mental Component Summary (PCS, MCS) Scores. PCS positively weights PF, RP, BP, GH and VT, whereas MCS positively weights VT, SF, RE and MH. MCIDs for SF-36 are defined as $\geq 5$. 0 -point increases from baseline in domain scores and $\geq 2.5$-point increases from baseline for PCS and MCS scores. ${ }^{1}$ 618 SF-36 domain scores were compared with US general population norms specifically matched to the age and gender distribution of the RAPID 2 trial population, ${ }^{1} 618$ and displayed as a 'spydergram'. 19

SF-36 was completed at baseline and weeks 12 and 24. All other PROs were assessed at baseline, and weeks 1, 2, 4, 6, 8, 12 and 24. Pain and physical function were also assessed at weeks 16 and 20.

Clinical efficacy assessments in RAPID 2 included the Disease Activity Score 28-joint count assessment-erythrocyte sedimentation rate (DAS28). ${ }^{5}$ Low disease activity (LDA) was defined as $\mathrm{DAS} 28 \leq 3.2$, and remission as DAS28 $\leq 2.6$. Radiographs of the hands and feet were assessed using the van der Heijde modified Total Sharp Score (mTSS). ${ }^{5}$ Non-progression was defined as a change from baseline in $\mathrm{mTSS} \leq 0.5$. Clinical and Simplified Disease Activity Indexes (CDAI and SDAI) were also calculated. ${ }^{20}$

\section{Statistical analyses}

\section{Response to treatment}

Analyses were conducted on the intent-to-treat (ITT) population, which included all randomised patients. Further details are provided in the online supplementary material.

Post hoc comparisons of the proportion of patients reporting improvements $\geq \mathrm{MCID}$ for each $\mathrm{PRO}$ were performed using repeated-measures logistic regression with region, treatment, time and treatment by time interactions as factors and baseline scores as covariates.

\section{NNT and correlations}

Additional post hoc analyses were performed to calculate the percentage of patients reporting improvements $\geq \mathrm{MCID}$ in up to six PROs (pain, PtGA, HAQ-DI, fatigue, SF-36 PCS and MCS scores) in the active treatment arms (CZP 200 or $400 \mathrm{mg}$ plus MTX) and placebo plus MTX group. ${ }^{21} 22$ NNT was assessed using the following formula: NNT $=1 /$ (response in active treatment - response in placebo group). Missing data were imputed using non-responder imputation.

Correlations between PROs at week 24 were analysed using Pearson correlations, and interpreted as $\geq-0.3$ to $\leq 0.3=$ low degree of correlation, $>-0.6$ to $<-0.3$ or $>0.3$ to $<0.6=\bmod -$ erate degree and -1.0 to $\leq-0.6$ or $\geq 0.6$ to $1.0=$ high degree. ${ }^{23}$ Correlations between PROs and DAS28, mTSS, CDAI and SDAI were also assessed.

\section{Predictive analyses}

To assess the predictive value of PROs on time to onset of response, two different analyses were performed. The first was based on achieving LDA at week 24 in patients who had improvements $\geq \mathrm{MCID}$ in pain at weeks 6 and 12 (week 6 responders), and those achieving these improvements at week 12 but not at week 6 (week 12 responders). The second analysis evaluated outcomes in patients reporting improvements $\geq \mathrm{MCID}$ in pain VAS at week 12 (pain week 12 responders) and those who did not (pain week 12 non-responders). As well as determination of LDA, DAS28 remission, achievement of patient acceptable symptom state (PASS, $\leq 1.04^{24}$ ) or normative HAQ-DI scores $\left(\leq 0.5^{25} 26\right)$ and rate of mTSS progression at week 24 were also assessed. Subjects without ACR20 responses at weeks 12 and 14 were obligatorily withdrawn at week 16 and were not included in the analyses. Responder status was defined using last observation carried forward (LOCF) imputation for LDA, DAS28 remission and HAQ-DI, and linear extrapolation for mTSS. Data from CZP 200 and $400 \mathrm{mg}$ groups were combined for analysis.

\section{RESULTS}

\section{Patients}

Of 619 randomised patients (ITT population), 13\% placebo, and $71 \%$ and $74 \%$ CZP 200 mg-treated and 400 mg-treated patients, respectively, completed the study. ${ }^{5}$ Mean age of the population was 52 years, $82 \%$ were female and mean disease duration was 6.2 years. The burden of RA was significant, evidenced by high baseline PRO scores (table 1). Patients also reported markedly lower SF-36 domain scores compared with a US normative population without arthritis. The impact of RA on HRQoL was particularly evident in PF, RP, BP, GH and RE domains.

\section{Impact of CZP on pain, PtGA, physical function, fatigue and HROOL}

CZP treatment was associated with reductions in pain, PtGA, HAQ-DI and fatigue as early as week 1 , which were statistically significant and clinically meaningful ( $\geq$ MCID) compared with placebo (see online supplementary figure S1). CZP-treated patients also reported significant improvements over placebo in overall HRQoL (SF-36) at the first postbaseline assessment at week 12 (figure S1).

Reductions in pain, PtGA, improvements in physical function, fatigue and HRQoL were maintained until the end of study at week $24(p<0.001)$ (figure 1). At week 24, significantly more patients in the CZP plus MTX groups reported improvements $\geq \mathrm{MCID}$ in all six PROs (pain, PtGA, physical function, fatigue and PCS and MCS scores) (figure $1 \mathrm{~F}$ ).

Improvements $\geq \mathrm{MCID}$ were evident in all eight SF-36 domain scores at week 12 for the CZP plus MTX groups, which were maintained until week 24 (figure 2), with the largest improvements at week 24 in the RP, BP and RE domains compared with baseline.

The beneficial effects of CZP were similar between 200 and $400 \mathrm{mg}$ groups with no statistical significance between groups in any PROs.

\section{Analysis of NNT and correlations between individual PROs at week 24 (ITT population)}

At week 24, 63\% of CZP $200 \mathrm{mg}$ plus MTX patients reported clinically meaningful improvements in one or more PRO compared with $13 \%$ in placebo. Approximately $23 \%$ CZP plus MTX-treated patients reported clinically meaningful improvements $(\geq \mathrm{MCID}$ ) in all six PROs compared with $3 \%$ in placebo.

NNT to achieve simultaneous clinically meaningful improvements in one, two, three or four of six PROs was approximately two additional patients, and NNT to achieve improvements in five of six PROs was three. NNT for improvements in all six PROs was approximately five patients (figure 3). Further analyses were conducted to assess which of the six PROs was driving the increase in NNT to five; indicating that patients were 
Table 1 Baseline PRO scores

\begin{tabular}{|c|c|c|c|}
\hline PRO (mean (SD)) & PBO+MTX (n=127) & $\begin{array}{l}\text { CZP } 200 \mathrm{mg}+\mathrm{MTX} \\
(\mathrm{n}=246)\end{array}$ & $\begin{array}{l}\text { CZP } 400 \mathrm{mg}+\mathrm{MTX} \\
(\mathrm{n}=246)\end{array}$ \\
\hline Pain VAS (range 0-100 mm) & $59.7(22.2)$ & $62.0(19.3)$ & $60.5(20.1)$ \\
\hline PtGA VAS (range 0-100 mm) & $59.7(21.9)$ & $62.5(20.3)$ & $61.0(19.6)$ \\
\hline HAQ-DI (range 0-3) & $1.6(0.6)$ & $1.6(0.6)$ & $1.6(0.6)$ \\
\hline Fatigue FAS NRS (range 0-10) & $6.5(1.8)$ & $6.7(1.9)$ & $6.4(1.8)$ \\
\hline \multicolumn{4}{|l|}{ SF-36 (range $0-100$ ) } \\
\hline PCS & $31.1(6.7)$ & $30.6(5.9)$ & $31.1(6.3)$ \\
\hline MCS & $40.1(11.4)$ & $38.6(10.9)$ & $39.6(10.9)$ \\
\hline \multicolumn{4}{|l|}{ Domains } \\
\hline Physical functioning & $34.5(21.4)$ & $30.5(20.0)$ & $32.9(20.0)$ \\
\hline Role physical & $16.8(27.8)$ & $12.3(24.4)$ & $14.2(25.7)$ \\
\hline Bodily pain & $30.8(16.9)$ & $29.2(14.3)$ & $30.8(14.7)$ \\
\hline General health & $34.4(15.8)$ & $34.7(15.5)$ & $34.3(14.7)$ \\
\hline Vitality & $36.9(18.7)$ & $36.0(17.7)$ & $38.1(17.2)$ \\
\hline Social functioning & $48.8(25.0)$ & $46.2(23.0)$ & $49.3(24.4)$ \\
\hline Role emotional & $38.0(41.6)$ & $27.1(39.3)$ & $31.4(38.7)$ \\
\hline Mental health & $53.5(19.3)$ & $52.1(18.1)$ & $52.9(18.2)$ \\
\hline
\end{tabular}

more likely to report clinically meaningful improvements in pain, PtGA, physical function, fatigue and SF-36 PCS than MCS. At week 24, of those CZP $200 \mathrm{mg}$ plus MTX patients with improvements $\geq \mathrm{MCID}$ in five of six PROs, $67 \%$ reported changes $\geq$ MCID in SF-36 PCS and $47 \%$ in SF-36 MCS scores. In contrast, the majority of patients in the $200 \mathrm{mg}$ group (95\%) reported clinically meaningful improvements in the remaining four PROs (pain 96\%; PtGA 98\%, HAQ-DI 96\%; fatigue 98\%). Similar results were observed in the CZP 400 plus MTX group.

To further explore the increase in the NNT to achieve improvements in all six PROs, correlation analyses between PROs and clinical parameters at week 24 were performed. These are presented for the CZP $200 \mathrm{mg}$ plus MTX dose group, and were similar in subjects receiving CZP $400 \mathrm{mg}$ plus MTX. As suggested by the NNT results, there were moderate to high correlations between pain, PtGA, HAQ-DI and fatigue; with the highest observed between pain and PtGA and fatigue, and the lowest between $\mathrm{HAQ}-\mathrm{DI}$ and fatigue (see online supplementary table S1). Correlations between SF-36 scores and other PROs were mostly moderate. High, negative correlations were observed between SF-36 BP domain scores and pain, VT and fatigue, and PF or PCS and HAQ-DI. MCS, RE and MH scores had the lowest negative correlations with HAQ-DI. No significant correlations were observed between change from baseline in PCS and MCS scores at week 24 ( $p=0.888)$. As shown in the NNT analyses, MCS and mental domain scores had lower correlations with the rest of the PROs; thus including MCS scores increased the NNT from two to three patients to almost six patients. There were low correlations between DAS28, and MCS and mental domain scores. Similarly, correlations between change from baseline in mTSS and the various PROs or between mTSS and DAS28 were low.

\section{Effect of time to onset of improvements in pain on clinical outcomes at week 24 (ITT population)}

As pain is considered to be the most important symptom of RA and because of the excellent correlations found between pain and the other PROs, improvements $\geq \mathrm{MCID}$ in pain were investigated to determine their potential to predict outcomes at week 24 . In an analysis of week 6 and 12 responders, approximately $27 \%$ of patients who reported improvements $\geq$ MCID in pain at week 6 achieved LDA at week 24 compared with $16 \%$ of patients who were week 12 but not week 6 responders.

Of the 492 patients included in the ITT population, 414 and 78 patients were classified as pain week 12 responders and nonresponders respectively. More pain week 12 responders than non-responders had LDA (20.0\% vs 3.9\%) and remission $(10.4 \%$ vs $1.3 \%$ ) at week 24 . Similarly, more week 12 responders had PASS (49.8\% vs $39.0 \%)$ and normative HAQ-DI scores $(23.4 \%$ vs $16.9 \%)$. The number of patients rated as mTSS non-progressors at week 24 was comparable between week 12 responders and non-responders (80.9\% vs $80.0 \%$ ). At week $24,77.1 \%$ and $46.2 \%$ of week 12 responders versus non-responders remained in the study respectively.

\section{DISCUSSION}

In this report, data from the RAPID 2 trial confirm that CZP provides broad relief of the multiple burdens imposed by RA. CZP was associated with rapid, clinically meaningful improvements in multiple PROs, observed as early as week 1 , and maintained until study end at 6 months. These results mirror those observed in the RAPID 1 trial, ${ }^{6}$ and data reported for other TNF inhibitors in randomised controlled trials, ${ }^{1}{ }^{18}{ }^{27-31}$ although the rapid onset of action in all PROs observed with CZP (by week 1) has not been previously reported with the other agents. ${ }^{1}$

To further demonstrate the benefits of CZP on PROs, improvements $\geq$ MCID in NNT and predictive analyses were used. Both methods can provide patients and physicians with meaningful information on treatment effect, such as how likely an individual patient is to benefit from treatment with CZP and whether the level of response within the first 12 weeks can determine longer term clinical outcomes.

To the authors' knowledge, this is the first study evaluating the benefit of an anti-TNF in RA by calculating the NNT using a variety of PROs. Previous work has investigated the NNT for other TNF inhibitors based on clinical responses such as ACR 20/50/70.32-39 One study of TNF inhibitors (etanercept, infliximab or adalimumab), analysing NNT according to HAQ-DI, reported an NNT of $1.94 .{ }^{37}$ In the present analysis, the NNT to achieve clinically meaningful improvements in up to five of six PROs was similarly low; only two to three additional patients needed to be treated to have at least one patient report 
A

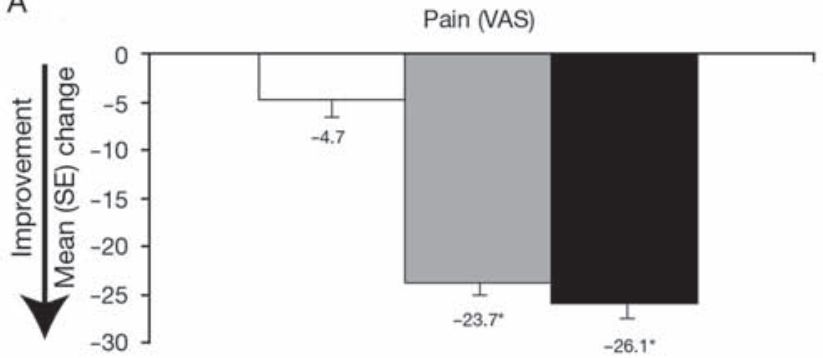

C

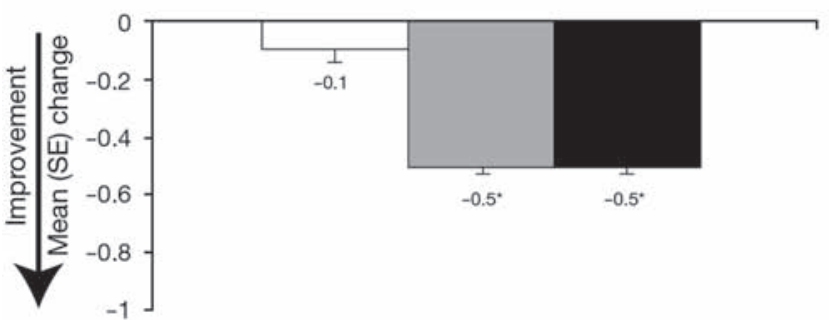

B

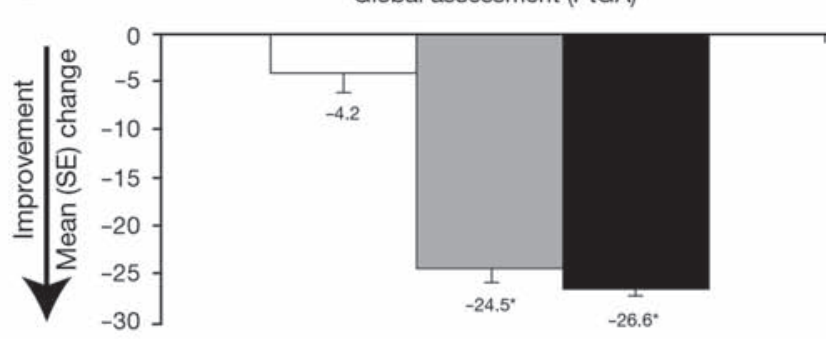

D

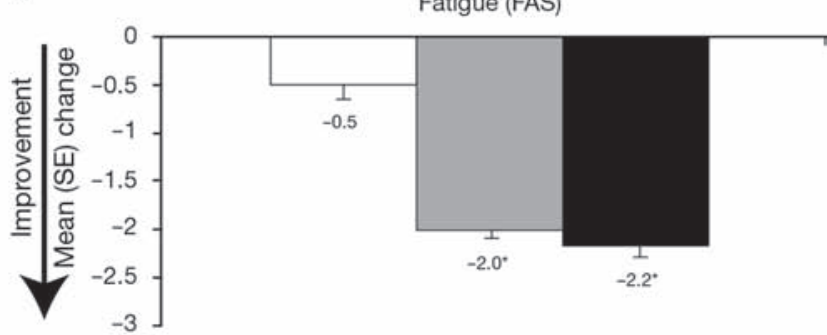

E
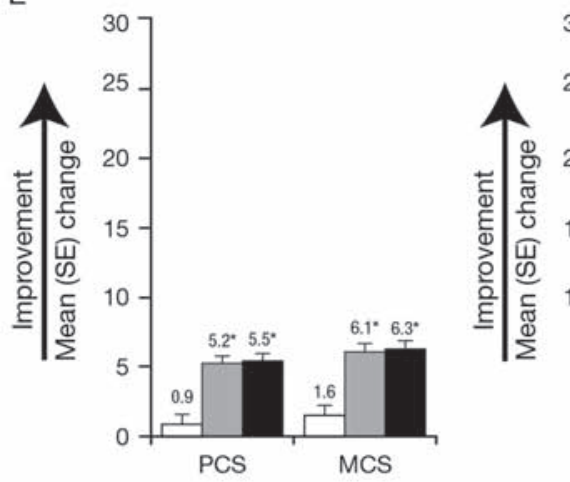

$\left.\begin{array}{l}30 \\ 25 \\ 20- \\ 15- \\ 10- \\ 5- \\ 0\end{array}\right]$

HRQoL (SF-36)

$\mathrm{F}$

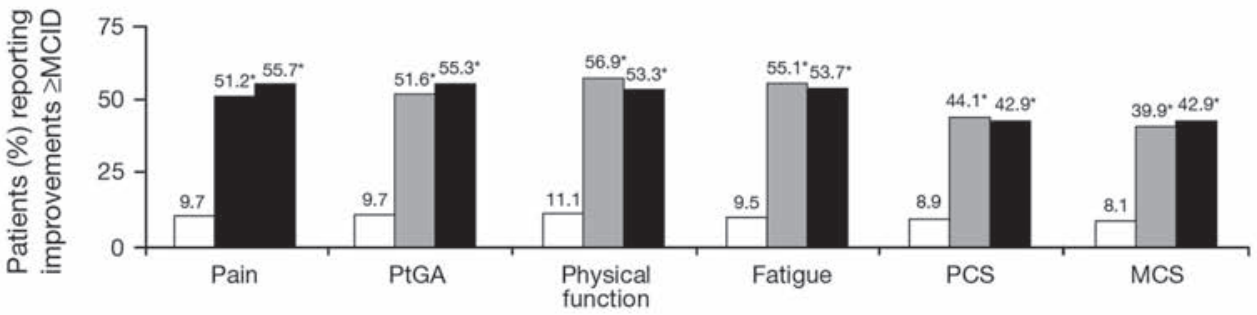

$\cdot p<0.001$ for CZP vs PBO

$P B O+\operatorname{MTX}(n=127)$

CZP $200 m g+\operatorname{MTX}(n=246)$

CZP $400 \mathrm{mg}+\operatorname{MTX}(n=246)$

Figure 1 Adjusted mean change from baseline (SE) and percentage of patients reporting improvements $\geq M C I D$ in pain, PtGA, physical function, fatigue and health-related quality of life at week 24 (ITT population, LOCF). ${ }^{*} p<0.001$ for the CZP groups vs placebo. BP, bodily pain; CZP, certolizumab pegol; $\mathrm{GH}$, general health; ITT, intent to treat; MCID, minimal clinically important difference; MCS, mental component summary; $\mathrm{MH}$, mental health; MTX, methotrexate; PB0, placebo; PCS, physical component summary; PF, physical functioning; PtGA, patient's global assessment of disease activity; RE, role emotional; RP, role physical; SF, social functioning; SF-36, short-form 36-item health survey; VT, vitality.

simultaneous relief in five PROs, and the NNT for improvements in all six PROs was five to six patients. Further analyses indicated that it was more difficult for patients to achieve improvements $\geq$ MCID in SF-36 PCS and more particularly MCS than in pain, PtGA, fatigue and physical function. This is not surprising as SF-36 measures additional aspects of the burden of $\mathrm{RA}$ and reporting improvements in complex concepts included in the mental component score implies a broader and holistic relief.
Further support for the use of all PRO measures including individual SF-36 domains to fully assess treatment benefit is provided by the correlation analyses. There were high correlations between pain, disease activity, fatigue and physical function; however, these improvements were less well correlated with those reported for HRQoL. As expected, correlations between mTSS and the PROs or other clinical parameters were low. Physical function scores (HAQ-DI) showed strong correlations with SF-36 PF and PCS scores as expected, but also BP domain 
A

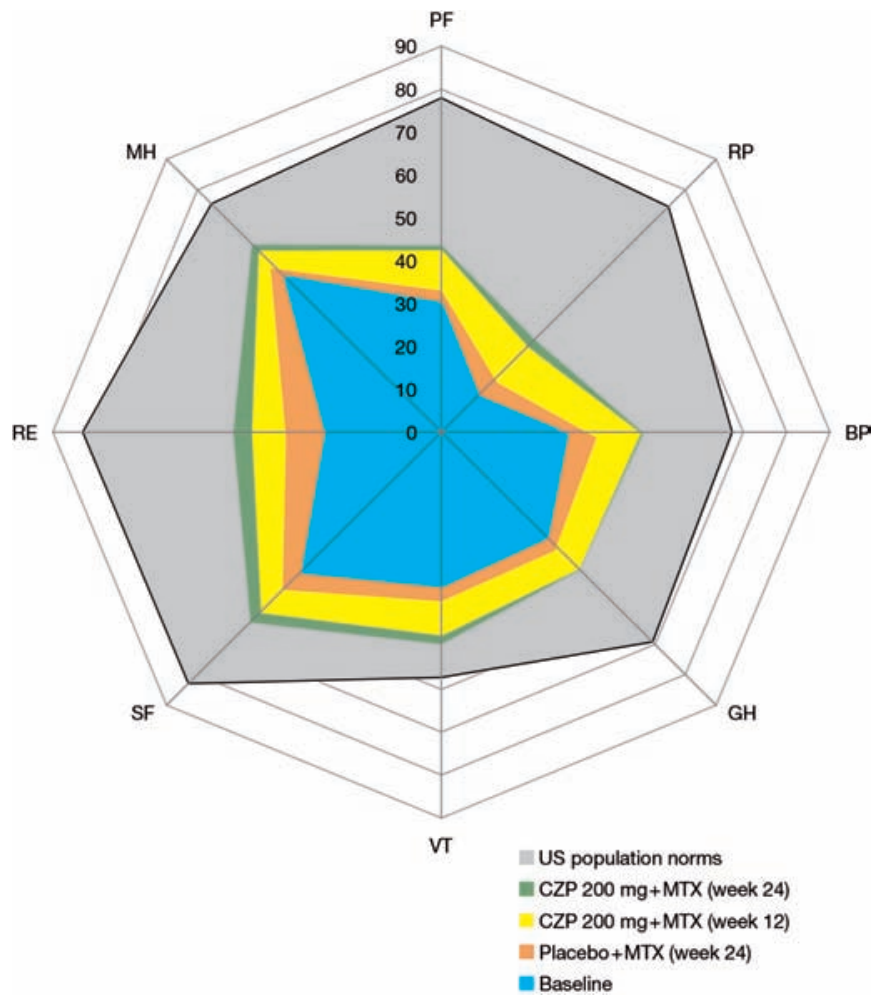

Figure 2 Improvements in short-form 36-item (SF-36) domain scores (ITT population, LOCF). A. Spydergram of SF-36 domains at baseline and following treatment with CZP $200 \mathrm{mg}$. Scores were plotted from 0 (worst) to 100 (best) with demarcations along axes of the domains indicating changes of 10 points representing one to two times MCID. B. Percentage of patients reporting improvements $\geq M C I D$ in SF-36 domains at week 24 ; ${ }^{*} p \leq 0.001 ; \dagger p \leq 0.01$. BP, bodily pain; CZP, certolizumab pegol; GH, general health; ITT, intent to treat; MCID, minimum clinically important difference; $\mathrm{MH}$, mental health; MTX, methotrexate; PF, physical functioning; RE, role emotional; RP, role physical; SF, social functioning; VT, vitality.

and only moderate correlations with fatigue (FAS) scores and low correlations with SF-36 domains assessing MH. High correlations were also observed between PF and BP, and between VT and $\mathrm{SF}$ and $\mathrm{MH}$. Interestingly, strong correlations were observed between certain 'physical' and 'mental' domains (RP and RE; $\mathrm{BP}$ and SF). Evaluating treatment-associated changes across all PROs is thus a better method for assessing change in multidimensional function. Careful evaluation of all SF-36 domain scores offers more information than the summary scores alone. For example, correlations between FAS and SF-36 MCS scores were lower than those between FAS and SF-36 VT domain scores. As VT is one of the domains scored positively when calculating MCS scores, these results indicate that the other three 'mental' domains (SF, and particularly MH and RE) provide the greatest contribution to changes in SF-36 MCS scores. FAS had high/marked correlations with pain VAS and PtGA followed by VT, which could indicate that FAS and SF-36 VT scores provide complementary assessments for measuring fatigue/tiredness; FAS may be more appropriate to evaluate physical aspects of fatigue, whereas SF-36 VT more fully assesses mental aspects such as pep, motivation and energy. Therefore, although SF-36 PCS and MCS scores provide a good summary metric of HRQoL, they do not fully reflect the overall magnitude of changes within individual domains. ${ }^{17} 19$ Physicians should consider data from all SF-36 domains as well as PCS and MCS scores in their decision-making process. To facilitate analysis and interpretation of treatment-related effects across the individual domains, 'spydergrams' were used to depict SF-36 results, which allowed a more global comparison of disease-related decrements in HRQoL versus normative data. ${ }^{19}$

A limitation of the NNT analysis is that patients with active disease in the placebo plus MTX group were used as the comparator and, therefore, the analysis only relates to this comparison. Nevertheless, the low NNT confirms the efficacy of CZP regarding reported improvements in $\mathrm{PROs}$, indicating that relatively few patients need to be treated with CZP to achieve relief from the burdens of RA.

More patients with responses by week 6 had LDA at week 24 than those with responses at week 12. Similarly, those patients achieving pain MCID by week 12 were more likely to have better 


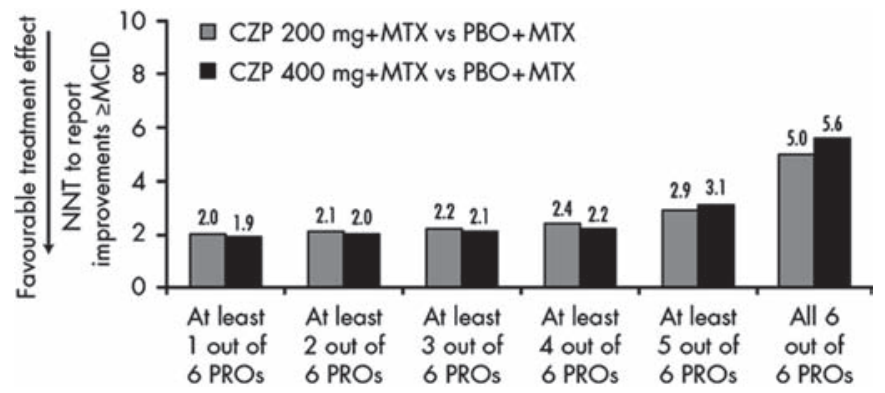

Figure 3 NNTs to report improvements $\geq M C I D s$ in one, two, three, four, five or six PROs after 24 weeks of treatment with CZP 200 mg+MTX and CZP $400 \mathrm{mg}$ plus MTX. CZP, certolizumab pegol; MCID, minimum clinically important difference; NNT, number needed to treat; MTX, methotrexate; PROs, patient-reported outcomes.

outcomes than those patients who did not achieve pain MCID at week 12, including LDA, remission and acceptable HAQ-DI scores. Taken together these results indicate that early improvement in PROs predict both clinical and PROs at 6 months. They complement previous analyses from the RAPID 1 trial demonstrating that patients who reported more rapid improvements in disease activity had higher chances of achieving better clinical outcomes at 1 year. ${ }^{40}$

Overall, these results suggest that patients treated with CZP who achieve early responses according to either PROs or LDA have a greater chance of avoiding long-term disability. Because of the short 6-month study duration of RAPID 2, longer term data (1-2 years) are needed to confirm the use of time to responses by PROs as a predictor of better clinical outcomes.

In conclusion, CZP 200 or $400 \mathrm{mg}$ plus MTX administered every 2 weeks provides broad relief from the multiple burdens of RA. Significant and clinically meaningful improvements, low NNTs and increased likelihood of achieving longer term outcomes with an earlier PRO response indicate that CZP is an effective treatment option, offering substantial benefits to patients.

Acknowledgements The authors thank Christophe Gengler, Phil Brown, Owen Davies and Lee Gervitz from UCB for their assistance with the data analyses and critical review of the manuscript. They acknowledge the editorial services of Andrew Richardson from PAREXEL, which were funded by UCB.

Funding The RAPID 2 study was fully funded by UCB.

Competing interests VS has worked as an independent biopharmaceutical consultant in clinical development and regulatory affairs since September 1991 and is currently a consultant to various sponsors including UCB, but has not and does not now hold stock in any company. JSS serves as a consultant to UCB. RFvV serves as a consultant to UCB and has received research funding from UCB. PM serves as a consultant to UCB, received research funding from UCB and has received an honorarium for speaking for UCB. GRB serves as a consultant to UCB and has received honoraria from UCB for speaking. FH has no competing interests. DK serves as a consultant to UCB. EN is a Business \& Decision Life Science consultant working for UCB. GC is a full-time employee of and holds stocks in UCB. MS serves as a consultant to UCB and has received research funding from UCB.

Ethics approval Institutional review boards or ethics committees approved the protocol at each centre. All patients gave written consent, and the study was conducted in accordance with the Declaration of Helsinki.

Provenance and peer review Not commissioned; externally peer reviewed.

\section{REFERENCES}

1. Strand V, Singh JA. Newer biological agents in rheumatoid arthritis: impact on health-related quality of life and productivity. Drugs 2010;70:121-45.

2. Osiri M, Suarez-Almazor ME, Wells GA, et al. Number needed to treat (NNT): implication in rheumatology clinical practice. Ann Rheum Dis 2003;62:316-21.

3. Keystone $\mathbf{E}$, Heijde D, Mason D Jr, et al. Certolizumab pegol plus methotrexate is significantly more effective than placebo plus methotrexate in active rheumatoid arthritis: findings of a fifty-two-week, phase III, multicenter, randomized, double-blind, placebo-controlled, parallel-group study. Arthritis Rheum 2008;58:3319-29.

4. Fleischmann $\mathbf{R}$, Vencovsky J, van Vollenhoven RF, et al. Efficacy and safety of certolizumab pegol monotherapy every 4 weeks in patients with rheumatoid arthritis failing previous disease-modifying antirheumatic therapy: the FAST4WARD study. Ann Rheum Dis 2009;68:805-11.

5. Smolen J, Landewé RB, Mease P, et al. Efficacy and safety of certolizumab pegol plus methotrexate in active rheumatoid arthritis: the RAPID 2 study. A randomised controlled trial. Ann Rheum Dis 2009;68:797-804.

6. Strand V, Mease P, Burmester GR, et al. Rapid and sustained improvements in healthrelated quality of life, fatigue, and other patient-reported outcomes in rheumatoid arthritis patients treated with certolizumab pegol plus methotrexate over 1 year: results from the RAPID 1 randomized controlled trial. Arthritis Res Ther 2009;11:R170.

7. Arnett FC, Edworthy SM, Bloch DA, et al. The American Rheumatism Association 1987 revised criteria for the classification of rheumatoid arthritis. Arthritis Rheum 1988;31:315-24

8. Wells GA, Tugwell P, Kraag GR, et al. Minimum important difference between patients with rheumatoid arthritis: the patient's perspective. J Rheumatol 1993;20:557-60.

9. Wells G, Li T, Maxwell L, et al. Determining the minimal clinically important differences in activity, fatigue, and sleep quality in patients with rheumatoid arthritis. J Rheumatol 2007;34:280-9.

10. Farrar JT, Young JP Jr, LaMoreaux L, et al. Clinical importance of changes in chronic pain intensity measured on an 11-point numerical pain rating scale. Pain 2001:94:149-58.

11. Dworkin RH, Turk DC, Wyrwich KW, et al. Interpreting the clinical importance of treatment outcomes in chronic pain clinical trials: IMMPACT recommendations. J Pain 2008;9:105-21.

12. Strand V, Bombardier C, Maetzel A. Use of minimum clinically important differences [MCID] in evaluating patient responses to treatment of RA. Arthritis Rheum 2001;44(Suppl):S187

13. Khanna D, Pope JE, Khanna PP, et al. The minimally important difference for the fatigue visual analog scale in patients with rheumatoid arthritis followed in an academic clinical practice. J Rheumatol 2008;35:2339-43.

14. Fries JF, Spitz P, Kraines RG, et al. Measurement of patient outcome in arthritis. Arthritis Rheum 1980;23:137-45.

15. Bruce B, Fries JF. The Stanford Health Assessment Questionnaire: dimensions and practical applications. Health Qual Life Outcomes 2003;1:20.

16. Tugwell P, Wells G, Strand V, et al. Clinical improvement as reflected in measures of function and health-related quality of life following treatment with leflunomide compared with methotrexate in patients with rheumatoid arthritis: sensitivity and relative efficiency to detect a treatment effect in a twelve-month, placebocontrolled trial. Leflunomide Rheumatoid Arthritis Investigators Group. Arthritis Rheum 2000;43:506-14

17. Ware JE, Kosinski M, Keller SK. SF-36 Physical and Mental Health Summary Scales: A User's Manual. Boston: New England Medical Center, The Health Institute, 1994.

18. Strand V, Singh JA. Improved health-related quality of life with effective diseasemodifying antirheumatic drugs: evidence from randomized controlled trials. Am J Manag Care 2008;14:234-54.

19. Strand V, Crawford B, Singh J, et al. Use of 'spydergrams' to present and interpret SF-36 health-related quality of life data across rheumatic diseases. Ann Rheum Dis 2009;68:1800-4.

20. Smolen JS, Breedveld FC, Schiff MH, et al. A simplified disease activity index for rheumatoid arthritis for use in clinical practice. Rheumatology (Oxford) 2003:42:244-57.

21. Khanna D, Yan X, Tashkin DP, et al. Impact of oral cyclophosphamide on healthrelated quality of life in patients with active scleroderma lung disease: results from the scleroderma lung study. Arthritis Rheum 2007;56:1676-84.

22. Moore RA, Moore OA, Derry S, et al. Responder analysis for pain relief and numbers needed to treat in a meta-analysis of etoricoxib osteoarthritis trials: bridging a gap between clinical trials and clinical practice. Ann Rheum Dis 2010;69:374-9.

23 Cohen J. Statistical Power Analysis for the Behavioral Sciences. 2nd edition. Hillsdale, $\mathrm{NJ}$ : Lawrence Erlbaum Associates 1988.

24. Heiberg T, Kvien TK, Mowinckel P, et al. Identification of disease activity and health status cut-off points for the symptom state acceptable to patients with rheumatoid arthritis. Ann Rheum Dis 2008;67:967-71.

25. Wells GA, Boers M, Shea B, et al. Minimal disease activity for rheumatoid arthritis: a preliminary definition. J Rheumato/ 2005:32:2016-24.

26. Pietrapertosa D, Salaffi F, Peluso G, et al. Residual minimal disease activity in rheumatoid arthritis: a simple definition through an in-depth statistical analysis of the major outcome. Rheumatology (Oxford) 2009;48:1242-6.

27. St Clair EW, van der Heijde DM, Smolen JS, et al. Combination of infliximab and methotrexate therapy for early rheumatoid arthritis: a randomized, controlled trial. Arthritis Rheum 2004;50:3432-43.

28. van der Kooij SM, de Vries-Bouwstra JK, Goekoop-Ruiterman YP, et al. Patientreported outcomes in a randomized trial comparing four different treatment strategies in recent-onset rheumatoid arthritis. Arthritis Rheum 2009;61:4-12. 
29. Breedveld FC, Weisman MH, Kavanaugh AF, et al. The PREMIER study: A multicenter, randomized, double-blind clinical trial of combination therapy with adalimumab plus methotrexate versus methotrexate alone or adalimumab alone in patients with early, aggressive rheumatoid arthritis who had not had previous methotrexate treatment. Arthritis Rheum 2006;54:26-37.

30. Kimel M, Cifaldi M, Chen N, et al. Adalimumab plus methotrexate improved SF-36 scores and reduced the effect of rheumatoid arthritis (RA) on work activity for patients with early RA. J Rheumatol 2008;35:206-15.

31. Kekow J, Moots RJ, Emery P, et al. Patient-reported outcomes improve with etanercept plus methotrexate in active early rheumatoid arthritis and the improvement is strongly associated with remission: the COMET trial. Ann Rheum Dis 2010;69:222-5

32. Blumenauer B, Judd M, Cranney A, et al. Etanercept for the treatment of rheumatoid arthritis. Cochrane Database Syst Rev 2003:4:CD004525.

33. Navarro-Sarabia F, Ariza-Ariza R, Hernández-Cruz B, et al. Adalimumab for treating rheumatoid arthritis. J Rheumatol 2006;33:1075-81.

34. Navarro-Sarabia F, Ariza-Ariza R, Hernandez-Cruz B, et al. Adalimumab for treating rheumatoid arthritis. Cochrane Database Syst Rev 2005;3:CD005113.

35. Kristensen LE, Christensen $\mathrm{R}$, Bliddal $\mathrm{H}$, et al. The number needed to treat for adalimumab, etanercept, and infliximab based on ACR50 response in three randomized controlled trials on established rheumatoid arthritis: a systematic literature review. Scand J Rheumatol 2007;36:411-17.

36. Alonso-Ruiz A, Pijoan JI, Ansuategui E, et al. Tumor necrosis factor alpha drugs in rheumatoid arthritis: systematic review and metaanalysis of efficacy and safety. BMC Musculoskelet Disord 2008:9:52.

37. Barra L, Pope JE, Payne M. Real-world anti-tumor necrosis factor treatment in rheumatoid arthritis, psoriatic arthritis, and ankylosing spondylitis: cost-effectiveness based on number needed to treat to improve health assessment questionnaire. J Rheumatol 2009;36:1421-8.

38. Singh JA, Noorbaloochi S, Singh G. Golimumab for rheumatoid arthritis. Cochrane Database Syst Rev 2010;1:CD008341.

39. Kristensen L, Jakobsen A, Bartels E, et al. The number needed to treat for second-generation biologics when treating established rheumatoid arthritis: a systematic quantitative review of randomized controlled trials. Scand J Rheumatol 2011:40:1-7.

40. Keystone E, Curtis JR, Fleischmann $\mathrm{R}$, et al. Rapid improvement in the signs and symptoms of rheumatoid arthritis following certolizumab pegol treatment predicts better long-term outcomes: post-hoc analysis of a randomized controlled trial. J Rheumatol 2010; (In Press). 